\title{
Pogitare
Enfermagem
}

\section{AVALIAÇÃO DA QUALIDADE DO CENTRO CIRÚRGICO NA ESTRUTURA, PROCESSO E RESULTADOS}

\author{
José Augusto Gomes ${ }^{1}$ (i) \\ Maria Manuela Martins ${ }^{2}$ (i) \\ Daisy Maria Rizatto Tronchin ${ }^{3}$ (1) \\ Carla Sílvia Fernandes ${ }^{2}$ (1)
}

\section{RESUMO}

Objetivos: avaliar a qualidade dos centros cirúrgicos em hospitais portugueses no que se refere à estrutura, processo e resultado.

Método: estudo quantitativo e transversal, realizado entre os meses de janeiro e maio de 2018. A amostra foi constituída por 1.019 profissionais, de 71 hospitais portugueses. Recorreu-se à utilização de três instrumentos distintos para medir a estrutura, o processo e os resultados da qualidade no centro cirúrgico: a Escala Indicadores de Estrutura no Bloco Operatório, a Escala de Processos de Qualidade Âssistencial no Bloco Operatório e a Escala para medir os Resultados de Qualidade no Bloco Operatório.

Resultados: os fatores "especificidades dos grupos profissionais", "fluxos de comunicação", "controle para a segurança" e "trabalho em equipe" foram considerados de alta qualidade.

Conclusão: a avaliacão da qualidade do centro cirúrgico nas categorias de estrutura, processo e resultado permite identificar lacunas que podem ser melhoradas e que exigem maior atenção.

DESCRITORES: Avaliação em Saúde; Salas Cirúrgicas; Gestão da Qualidade; Enfermagem; Melhoria de Qualidade.

\section{EVALUACIÓN DE LA CALIDAD DEL CENTRO QUIRÚRGICO EN RELACIÓN CON LA ESTRUCTURA, EL PROCESO Y LOS RESULTADOS}

\section{RESUMEN:}

Objetivos: evaluar la calidad de los centros quirúrgicos en hospitales de Portugal en relación con la estructura, el proceso y el resultado. Método: estudio cuantitativo y transversal, realizado entre los meses de enero y mayo de 2018. La muestra estuvo compuesta por 1.019 profesionales de 71 hospitales de Portugal. Se recurrió al uso de tres instrumentos distintos para medir la estructura, el proceso y los resultados de la calidad en el centro quirúrgico, a saber: Escala de Indicadores de Estructura en la Sala Quirúrgica, Escala de Procesos de Calidad Asistencial en la Sala Quirúrgica y Escala para medir los Resultados de Calidad en la la Sala Quirúrgica. Resultados: los factores "especificidades de los grupos profesionales", "flujos de comunicación", "control para la seguridad" y "trabajo en equipo" se consideraron como de alta calidad. Conclusión: la evaluación de la calidad del centro quirúrgico en las categorías de estructura, proceso y resultado permite identificar deficiencias que pueden mejorarse y que exigen mayor atención.

DESCRIPTORES: Evaluación en Salud; Salas Quirúrgicas; Gestión de la Calidad; Enfermería; Mejora de la Calidad. 
A cirurgia mudou drasticamente nas últimas décadas; segurança, qualidade e eficiência tornaram-se os objetivos prioritários dos cuidados cirúrgicos do século ${ }^{(1-2)}$. $O$ centro cirúrgico é uma das estruturas mais complexas do sistema hospitalar, com um elevado grau de complexidade de equipamentos e procedimentos, pela sua multidisciplinariedade e pela sua intersecção com os vários setores do hospital ${ }^{(3)}$.

O centro cirúrgico é muitas vezes o maior contribuinte para o sucesso financeiro de um hospital, no entanto, é também uma das mais importantes unidades nesse ambiente, pelos elevados custos associados ${ }^{(4-5)}$. Por isso, necessita de uma gestão adequada que conduza à melhor eficiência e eficácia, mantendo ou desejavelmente melhorando os atuais níveis de qualidade assistencial(6). Numerosos estudos têm vindo a salientar a importância da utilização de indicadores de qualidade no centro cirúrgico, monitorizando a qualidade e segurança dos cuidados ${ }^{(7-10)}$.

Um dos principais desafios na avaliação da qualidade consiste em identificar quais os indicadores relevantes a serem incluídos. O trabalho de Donabedian permanece significativo para o que é hoje um movimento internacional da qualidade dos cuidados de saúde, integrando os conceitos centrais de estrutura, processo e resultado, para medir e melhorar a qualidade em saúde ${ }^{(11)}$. Donabedian esclarece que estas categorias não devem ser confundidas com atributos de qualidade, mas são classificações importantes para os tipos de informações que podem ser obtidas, a fim de inferir se a qualidade do atendimento é adequada ${ }^{(12)}$. Esses conceitos continuam sendo a base da avaliação da qualidade atual(13). Exemplo disso são as diretrizes da Organização Mundial de Saúde (OMS) para a Cirurgia Segura, que propõem o modelo de Donabedian de medição de prestação de cuidados de saúde ${ }^{(14)}$.

Apesar da especial importância que tem sido dado à qualidade em saúde nos últimos anos, no âmbito do centro cirúrgico, esta preocupação recai sobre as recomendações associadas à segurança dos cuidados.

Recorda-se que todos os residentes em Portugal têm acesso a cuidados de saúde prestados pelo Serviço Nacional de Saúde (NHS), financiados principalmente por meio de impostos. A prestação de cuidados de saúde é feita por serviços de saúde públicos e privados $^{(15)}$. Em Portugal, existem quatro níveis de cuidados à saúde: 1) primários (voltados à comunidade), 2) secundários (em unidades hospitalares), 3) cuidados pós-hospitalares no processo de reabilitação e 4) cuidados paliativos ${ }^{(16)}$. As reformas implementadas desde 2011 pelo Ministério da Saúde incluem, entre outras, o fortalecimento dos cuidados hospitalares ${ }^{(15)}$.

A rede hospitalar em Portugal sofreu um conjunto de importantes alterações nos últimos 40 anos, não só em termos de vínculo institucional como também no seu estatuto jurídico ${ }^{(17)}$. Estas reformas são visíveis na rede de cuidados disponíveis. Em 2014, Portugal contava com 225 hospitais, dos quais 113 pertenciam ao Sistema Nacional de Saúde público, tendo ao longo dos últimos anos havido fusões entre hospitais do setor público(15).

Atualmente, no universo do Sistema Nacional de Saúde público de Portugal, existem cerca de 170 centros cirúrgicos, com 569 salas cirúrgicas, distribuídos por 45 entidades e 82 unidades hospitalares. Á otimização da qualidade e eficiência da sala de cirurgia é uma chave crítica para o sucesso; nesse sentido, a avaliação assume um papel importante.

A avaliação da qualidade é cada vez mais reconhecida como um fator importante na melhoria da qualidade dos cuidados de saúde, principalmente nos centros cirúrgicos. Assim, este estudo tem como objetivo avaliar a qualidade assistencial no bloco operatório em hospitais portugueses no que se refere à sua estrutura, processo e resultado. 
Estudo quantitativo e transversal. Os questionários foram remetidos para 174 hospitais e respondidos por 71 (40,8\%). A amostra por conveniência contou com 1019 profissionais com atividade no centro cirúrgico. Foram considerados elegíveis para este estudo médicos e enfermeiros com atividade no bloco operatório, com atuação direta nos cuidados há pelo menos dois anos, dispostos a participar do estudo.

Os instrumentos de coleta de dados foram: um questionário sociodemográfico; três escalas referentes à avaliação da Estrutura, Processo e Resultado (Indicadores de Estrutura no Bloco Operatório - Escala IEBO, Processos de Qualidade Assistencial no Bloco Operatório - Escala PQABO, e Resultados de Qualidade no Bloco Operatório - Escala RQBO). O preenchimento dos instrumentos foi realizado por meio de um questionário eletrónico remetido para os conselhos de administração dos hospitais, que reencaminharam para os profissionais em causa. A pesquisa foi realizada entre os meses de janeiro e maio de 2018.

Os 28 itens da escala Indicadores de Estrutura no Bloco Operatório (IEBO) são agrupados em sete dimensões: ambiente e equipamentos (cinco itens), recursos para a qualidade e segurança (sete itens), circuitos no bloco operatório (quatro itens), instalações e requisitos de funcionamento (seis itens), formação e práxis no bloco operatório (três itens), continuidade na assistência de enfermagem (dois itens) e especificidades dos grupos profissionais (um item) ${ }^{(17)}$. As questões da escala englobam variáveis associadas às instalações (dimensões, infraestruturas, circuitos), ambiente (temperatura, umidade), equipamento (tecnologia, manutenção), recursos humanos (rácios, horários), financeiros e organizacionais (formação, ensino, etc.).

Quadro 1 - Critérios da escala Indicadores de Estrutura no Bloco Operatório - IEBO. Porto, Portugal, 2019 (continua)

\begin{tabular}{|c|c|c|c|}
\hline FATORES & MIN_MAX & $\begin{array}{c}\text { NÍVEL DE } \\
\text { QUALIDADE }\end{array}$ & VALORES \\
\hline \multirow{3}{*}{ Ambiente e equipamentos } & \multirow{3}{*}{$5-25$} & Baixa & 5 a 14 \\
\hline & & Média & 15 a 19 \\
\hline & & Alta & 20 a 25 \\
\hline \multirow{3}{*}{ Recursos para a qualidade e segurança } & \multirow{3}{*}{$7-35$} & Baixa & 7 a 20 \\
\hline & & Média & 21 a 27 \\
\hline & & Alta & 28 a 35 \\
\hline \multirow{3}{*}{ Circuitos no bloco operatório } & \multirow{3}{*}{$4-20$} & Baixa & 4 a 11 \\
\hline & & Média & 12 a 15 \\
\hline & & Alta & 16 a 20 \\
\hline \multirow{3}{*}{ Instalações e requisitos de funcionamento } & \multirow{3}{*}{$6-30$} & Baixa & 6 a 17 \\
\hline & & Média & 18 a 23 \\
\hline & & Alta & 24 a 30 \\
\hline \multirow{3}{*}{ Formação e práxis no bloco operatório } & \multirow{3}{*}{$3-15$} & Baixa & 3 a 8 \\
\hline & & Média & 9 a 11 \\
\hline & & Alta & 12 a 15 \\
\hline
\end{tabular}




\begin{tabular}{|l|c|c|c|}
\hline \multirow{2}{*}{ Continuidade na assistência de enfermagem } & \multirow{2}{*}{$2-10$} & Baixa & 2 a 5 \\
\cline { 3 - 4 } & & Média & 6 a 7 \\
\hline \multirow{2}{*}{ Especificidades dos grupos profissionais } & \multirow{2}{*}{$1-5$} & Alta & 8 a 10 \\
\cline { 3 - 4 } & \multirow{2}{*}{$1-5$} & Baixa & 1 a 2 \\
\cline { 3 - 4 } & & Média & 3 \\
\hline
\end{tabular}

Fonte: Os autores (2019)

A escala Processos de Qualidade Assistencial no Bloco Operatório (PQABO) é composta por 17 itens, validada para a população portuguesa e composta por quatro fatores: fluxos de comunicação (sete itens); estratégias de suporte aos processos de trabalho assistencial (seis itens); controle para a segurança (dois itens); e trabalho em equipe (dois itens) $)^{(18)}$. As questões da escala englobam variáveis associadas ao trabalho em equipe (relações interpessoais, liderança), comunicação, registos, know-how dos profissionais, utilização de checklist, briefing e debriefing, entre outros.

Quadro 2 - Critérios da escala Processos de Qualidade Assistencial no Bloco Operatório - PQABO. Porto, Portugal, 2019

\begin{tabular}{|c|c|c|c|}
\hline FATORES & MIN_MAX & $\begin{array}{l}\text { NÍVEL DE } \\
\text { QUALIDADE }\end{array}$ & VALORES \\
\hline \multirow{3}{*}{ Fluxos de comunicação } & \multirow{3}{*}{$7-35$} & Baixa & 7 a 20 \\
\hline & & Média & 21 a 27 \\
\hline & & Alta & 28 a 35 \\
\hline \multirow{3}{*}{$\begin{array}{l}\text { Estratégias de suporte aos processos de trabalho } \\
\text { assistencial }\end{array}$} & \multirow{3}{*}{$6-30$} & Baixa & 6 a 17 \\
\hline & & Média & 18 a 23 \\
\hline & & Alta & 24 a 30 \\
\hline \multirow{3}{*}{ Controle para a segurança } & \multirow{3}{*}{$2-10$} & Baixa & 2 a 5 \\
\hline & & Média & 6 a 7 \\
\hline & & Alta & 8 a 10 \\
\hline \multirow{3}{*}{ Trabalho em equipe } & \multirow{3}{*}{$2-10$} & Baixa & 2 a 5 \\
\hline & & Média & 6 a 7 \\
\hline & & Alta & 8 a 10 \\
\hline
\end{tabular}

Fonte: Os autores (2019)

A escala Resultados de Qualidade no Bloco Operatório (RAQBO) contem 13 itens e é composta por três fatores: avaliação da qualidade dos cuidados, avaliação dos processos e sistemas de controle ${ }^{(19)}$. As questões englobam variáveis associadas aos instrumentos 
de avaliação (indicadores, satisfação, número de complicações), avaliação de recursos humanos, de processos e resultados.

Quadro 3 - Critérios da escala Resultados de Qualidade no Bloco Operatório - RAQBO. Porto, Portugal, 2019

\begin{tabular}{|l|c|c|c|}
\hline FATORES & MIN_MAX & $\begin{array}{c}\text { NÍVEL DE } \\
\text { QUALIDADE }\end{array}$ & VALORES \\
\hline \multirow{2}{*}{ Avaliação da qualidade dos cuidados } & & Baixa & 6 a 17 \\
\hline \multirow{2}{*}{ Avaliação dos processos } & \multirow{2}{*}{ Jun-30 } & Média & 18 a 23 \\
\cline { 3 - 4 } & & Alta & 24 a 30 \\
\hline \multirow{2}{*}{ Sistemas de controle } & \multirow{2}{*}{ Apr-20 } & Baixa & 4 a 11 \\
\cline { 3 - 4 } & & Média & 12 a 15 \\
\cline { 3 - 4 } & & Alta & 16 a 20 \\
\hline
\end{tabular}

Fonte: Os autores (2019)

Nas três escalas, os itens são compostos por uma escala likert contendo cinco opções de resposta: "discordo totalmente", "discordo parcialmente", "indiferente", "concordo parcialmente" e "concordo totalmente". Para o tratamento dos dados, recorreu-se ao software Statistical Package for Social Sciences (SPSS v.24). Na análise dos dados, utilizou-se a estatística descritiva e inferencial, com medidas de tendência central e de dispersão. Para investigar a associação entre os itens pesquisados, foram utilizados testes não paramétricos, designadamente o teste de Kruskal-Wallis, adotando-se o intervalo de confiança de $95 \%$, com p-valor $<0,05$.

O projeto de pesquisa foi aprovado pelo comité de ética de uma unidade hospitalar $\left(N^{\circ}\right.$ CES246-16) e depois remetido para as outras instituições. Ao longo das diferentes etapas, foi garantido o anonimato e a confidencialidade dos dados.

\section{RESULTADOS}

A amostra foi composta por 1.019 participantes, dos quais $747(73,4 \%)$ são enfermeiros, e 271 (26,6\%) médicos. O sexo feminino prevaleceu com 702 (69,2\%); a idade dos participantes variou entre 24 e 68 anos (média=43 DP=7). No que se refere ao tempo de exercício profissional no bloco operatório, este variou entre 2 e 42 anos (média=14,6 $\mathrm{DP}=9,1)$.

Foram identificados 15 tipos de centro cirúrgico de acordo com o tipo de intervenção. Considerando o exercício profissional, 879 profissionais trabalham em centro cirúrgico 
central (todo o tipo de intervenção) (86,3\%), seguindo-se o bloco ambulatório 68 (6,7\%). Os restantes centros cirúrgicos têm pouca expressão (ortopedia $n=28$, pediatria dois, oftalmologia quatro, cardiotorácica cinco, otorrinolaringologia quatro, Estomatologia um, urologia um, neurocirurgia dois, periférico (bloco de periferia) dois, bloco de exames um, bloco de partos cinco e bloco de ginecologia/obstetrícia quatro.

No que se refere à distribuição regional dos profissionais, predominam os profissionais da região Norte 453 (44,5\%), seguindo-se a região Centro 239 (23,5\%), Lisboa e Vale do Tejo 188 (4\%), Alentejo 46 (4,5\%), Algarve 12 (1,2\%), Região Autónoma (RA) da Madeira três $(0,3 \%)$ e Região Autónoma (RA) Açores 19 (1,9\%), existindo ainda 59 profissionais $(5,8 \%)$ cuja região é indefinida.

$\mathrm{Na}$ Tabela 1, são apresentados os resultados das medidas descritivas resultantes da aplicação dos instrumentos de avaliação da qualidade no centro cirúrgico.

Tabela 1 - Caracterização do nível de Qualidade por dimensão e fator. Porto, Portugal, 2019

Fatores

Medidas descritivas

Mín Máx Mediana Moda Média DP* $\begin{gathered}\text { Nível de } \\ \text { qualidade }\end{gathered}$

Dimensão Estrutura

\begin{tabular}{llllllll}
\hline Ambiente e equipamentos & 5 & 25 & 19 & 20 & 17,9 & 4,7 & Média \\
\hline $\begin{array}{l}\text { Recursos para a qualidade e } \\
\text { segurança }\end{array}$ & 8 & 35 & 26 & 28 & 25 & 5,6 & Média \\
\hline Circuitos no bloco operatório & 4 & 20 & 12 & 4 & 11,6 & 4,8 & Média \\
\hline $\begin{array}{l}\text { Instalações e requisitos de } \\
\text { funcionamento }\end{array}$ & 7 & 30 & 21 & 22 & 20,4 & 5,9 & Média \\
\hline $\begin{array}{l}\text { Formação e práxis no bloco } \\
\text { operatório }\end{array}$ & 3 & 15 & 12 & 12 & 11,2 & 2,9 & Média \\
\hline $\begin{array}{l}\text { Continuidade na assistência de } \\
\text { enfermagem }\end{array}$ & 2 & 10 & 2 & 2 & 4 & 2,7 & Baixa \\
\hline $\begin{array}{l}\text { Especificidades dos grupos } \\
\text { profissionais }\end{array}$ & 1 & 5 & 4 & 4 & 3,9 & 1,1 & Alta \\
\hline
\end{tabular}

Dimensão Processo

\begin{tabular}{llllllll}
\hline Fluxos de comunicação & 8 & 35 & 29 & 30 & 28 & 4,7 & Alta \\
\hline $\begin{array}{l}\text { Estratégias de suporte aos } \\
\text { processos de trabalho assistencial }\end{array}$ & 6 & 30 & 21 & 22 & 20 & 5,1 & Média \\
\hline Controle para a segurança & 2 & 10 & 10 & 10 & 9 & 1,4 & Alta \\
\hline Trabalho em equipe & 2 & 10 & 9 & 10 & 9 & 1,5 & Alta \\
\hline Dimensão Resultado & 6 & 30 & 19 & 18 & 18,4 & 6,4 & Média \\
\hline $\begin{array}{l}\text { Avaliação da qualidade dos } \\
\text { cuidados }\end{array}$ & 4 & 20 & 12 & 12 & 12 & 4,1 & Média \\
\hline Avaliação dos processos & 3 & 15 & 10 & 10 & 10,1 & 3,3 & Média
\end{tabular}

*DP - Desvio Padrão

Fonte: Os autores (2019) 
$\mathrm{Na}$ Tabela 2, é apresentado o resultado das comparações das dimensões e fatores das escalas com o tipo de centro cirúrgico. Na avaliação, distinguiu-se o Bloco central, o Bloco da urgência, o Bloco ambulatório e o Outro bloco que integra os blocos de ortopedia, pediatria, oftalmologia, cardiotorácica, otorrinolaringologia, estomatologia, urologia, neurocirurgia, periférico, bloco de exames, bloco de partos e ginecologia/obstetrícia.

Tabela 2 - Comparações da qualidade por tipo de centro cirúrgico. Porto, Portugal, 2019 (continua)

\section{Dimensão Estrutura}

\begin{tabular}{|c|c|c|c|c|}
\hline \multirow[t]{2}{*}{ Fatores } & \multicolumn{2}{|c|}{ Valores da Escala } & \multicolumn{2}{|c|}{ Tipo de centro cirúrgico } \\
\hline & Média & $\begin{array}{l}\text { Desvio } \\
\text { Padrão } \\
\end{array}$ & $\begin{array}{l}\text { Estat. } \\
\text { teste }\end{array}$ & Valor-p \\
\hline \multicolumn{5}{|c|}{ Ambiente e equipamentos } \\
\hline Bloco central & 17,8 & 4,7 & \multirow[t]{5}{*}{32,5} & \multirow[t]{5}{*}{$<0,001$} \\
\hline Bloco da urgência & 12,4 & 3,2 & & \\
\hline Bloco ambulatório & 19,3 & 4,6 & & \\
\hline Bloco Ortopedia & 21,3 & 3,8 & & \\
\hline Outro bloco & 18,1 & 4,5 & & \\
\hline \multicolumn{5}{|c|}{ Recursos para a qualidade e segurança } \\
\hline Bloco central & 24.8 & 5,5 & \multirow[t]{5}{*}{36,1} & \multirow[t]{5}{*}{$<0,001$} \\
\hline Bloco da urgência & 18,9 & 4,4 & & \\
\hline Bloco ambulatório & 27,5 & 5,3 & & \\
\hline Bloco Ortopedia & 29,4 & 4,7 & & \\
\hline Outro bloco & 24,3 & 5,9 & & \\
\hline \multicolumn{5}{|c|}{ Circuitos no bloco operatório } \\
\hline Bloco central & 11,5 & 4,7 & \multirow[t]{5}{*}{23,1} & \multirow[t]{5}{*}{$<0,001$} \\
\hline Bloco da urgência & 9,3 & 3,6 & & \\
\hline Bloco ambulatório & 14 & 4,6 & & \\
\hline Bloco Ortopedia & 14,4 & 4,3 & & \\
\hline Outro bloco & 7,1 & 3,9 & & \\
\hline \multicolumn{5}{|c|}{ Instalações e requisitos de funcionamento } \\
\hline Bloco central & 20,3 & 5,7 & \multirow[t]{5}{*}{27,2} & \multirow[t]{5}{*}{$<0,001$} \\
\hline Bloco da urgência & 13,6 & 4,3 & & \\
\hline Bloco ambulatório & 22,5 & 5,2 & & \\
\hline Bloco Ortopedia & 26 & 6,2 & & \\
\hline Outro bloco & 16,4 & 6,7 & & \\
\hline \multicolumn{5}{|c|}{ Formação e práxis no bloco operatório } \\
\hline Bloco central & 11,2 & 2,8 & \multirow[t]{3}{*}{21,4} & \multirow[t]{3}{*}{$<0,001$} \\
\hline Bloco da urgência & 6,9 & 3,1 & & \\
\hline Bloco ambulatório & 11,8 & 2,5 & & \\
\hline
\end{tabular}




\begin{tabular}{|c|c|c|c|c|}
\hline Bloco Ortopedia & 12,8 & 2,3 & & \\
\hline Outro bloco & 9,8 & 2,9 & & \\
\hline \multicolumn{5}{|c|}{ Continuidade na assistência de enfermagem } \\
\hline Bloco central & 3,9 & 3,6 & 22,1 & $<0,001$ \\
\hline Bloco da urgência & 2,8 & 1,7 & & \\
\hline Bloco ambulatório & 5,3 & 2,9 & & \\
\hline Bloco Ortopedia & 5,4 & 2,9 & & \\
\hline Outro bloco & 3,9 & 2,5 & & \\
\hline \multicolumn{5}{|c|}{ Especificidades dos grupos profissionais } \\
\hline Bloco central & 3,9 & 1,1 & 2,3 & 0,517 \\
\hline Bloco da urgência & 4,2 & 0,8 & & \\
\hline Bloco ambulatório & 3,8 & 1,1 & & \\
\hline Bloco Ortopedia & 3,6 & 1,4 & & \\
\hline Outro bloco & 3,9 & 1 & & \\
\hline
\end{tabular}

\section{Dimensão Processo}

Fluxos de comunicação

\begin{tabular}{|c|c|c|c|c|}
\hline Bloco central & 28,8 & 4,6 & 12,3 & 0,007 \\
\hline Bloco da urgência & 24,2 & 5,6 & & \\
\hline Bloco ambulatório & 28,9 & 4,8 & & \\
\hline Bloco Ortopedia & 31,5 & 3,5 & & \\
\hline Outro bloco & 26,5 & 5,5 & & \\
\hline \multicolumn{5}{|c|}{ Estratégias de suporte aos processos de trabalho assistencial } \\
\hline Bloco central & 20,3 & 5 & 24,5 & $<0,001$ \\
\hline Bloco da urgência & 16,5 & 5,9 & & \\
\hline Bloco ambulatório & 22,8 & 5 & & \\
\hline Bloco Ortopedia & 24,3 & 5,1 & & \\
\hline Outro bloco & 18,9 & 5,1 & & \\
\hline \multicolumn{5}{|c|}{ Controle para a segurança } \\
\hline Bloco central & 9,3 & 1,4 & 4 & 0,258 \\
\hline Bloco da urgência & 9,6 & 0,7 & & \\
\hline Bloco ambulatório & 9,4 & 1,2 & & \\
\hline Bloco Ortopedia & 8,8 & 1,7 & & \\
\hline Outro bloco & 8,5 & 2,5 & & \\
\hline \multicolumn{5}{|l|}{ Trabalho em equipe } \\
\hline Bloco central & 8,6 & 1,5 & 4,7 & 0,196 \\
\hline Bloco da urgência & 8,2 & 1 & & \\
\hline Bloco ambulatório & 8,9 & 1,3 & & \\
\hline Bloco Ortopedia & 8,6 & 1,9 & & \\
\hline Outro bloco & 8,5 & 1,4 & & \\
\hline
\end{tabular}


Avaliação da qualidade dos cuidados

\begin{tabular}{|c|c|c|c|c|}
\hline Bloco central & 18,3 & 6,4 & 24,7 & $<0,001$ \\
\hline Bloco da urgência & 12,8 & 5,4 & & \\
\hline Bloco ambulatório & 21,3 & 5 & & \\
\hline Bloco Ortopedia & 23,2 & 6,4 & & \\
\hline Outro bloco & 15,5 & 5,6 & & \\
\hline \multicolumn{5}{|c|}{ Avaliação dos processos } \\
\hline Bloco central & 11,8 & 4 & 19,7 & 0,000 \\
\hline Bloco da urgência & 9,6 & 3,8 & & \\
\hline Bloco ambulatório & 13,8 & 3,9 & & \\
\hline Bloco Ortopedia & 15 & 4,2 & & \\
\hline Outro bloco & 9,9 & 3,4 & & \\
\hline \multicolumn{5}{|l|}{ Sistemas de controle } \\
\hline Bloco central & 9,9 & 3,2 & 31 & $<0,001$ \\
\hline Bloco da urgência & 6,2 & 2,8 & & \\
\hline Bloco ambulatório & 11,5 & 3,2 & & \\
\hline Bloco Ortopedia & 12 & 3,3 & & \\
\hline Outro bloco & 9,1 & 3,1 & & \\
\hline
\end{tabular}

Fonte: Os autores (2019)

Na Tabela 3 foi apresentado o resultado das comparações das dimensões e fatores das escalas com a região do país, distinguindo-se as regiões Norte, Centro, Lisboa e Vale do Tejo, Alentejo, Algarve, Madeira e Açores.

Tabela 3 - Comparações da qualidade por região. Porto, Portugal, 2019 (continua)

\section{Dimensão Estrutura}

\begin{tabular}{lcccc}
\hline \multirow{2}{*}{ Fatores } & \multicolumn{2}{c}{ Valores da Escala } & \multicolumn{2}{c}{ Por Região } \\
\cline { 2 - 4 } & Média & $\begin{array}{c}\text { Desvio } \\
\text { Padrão }\end{array}$ & $\begin{array}{c}\text { Estat. } \\
\text { teste }\end{array}$ & Valor-p \\
\hline Ambiente e equipamentos & 46,1 & $<0,001$ \\
\hline Norte & 17,9 & 4,7 & \\
\hline Centro & 17,4 & 4,6 \\
\hline Lisboa Vale do Tejo & 17,9 & 4,9 \\
\hline Alentejo & 20,5 & 3,1 \\
\hline Algarve & 13,4 & 5,9 \\
\hline Madeira & 20,3 & 0,6 \\
\hline Açores & 14,5 & 4,7 & \\
\hline
\end{tabular}


Recursos para a qualidade e segurança

$32,5<0,001$

\begin{tabular}{lcc}
\hline Norte & 25,7 & 5,6 \\
\hline Centro & 23,8 & 5,3 \\
\hline Lisboa Vale do Tejo & 24,9 & 5,7 \\
\hline Alentejo & 24,4 & 4,2 \\
\hline Algarve & 18,6 & 7,3 \\
\hline Madeira & 26 & 3,6 \\
\hline Açores & 25,1 & 3,9 \\
\hline
\end{tabular}

Circuitos no bloco operatório

\begin{tabular}{|c|c|c|c|c|}
\hline Norte & 11,6 & 4,9 & & \\
\hline Centro & 12,3 & 4,4 & & \\
\hline Lisboa Vale do Tejo & 11,4 & 4,9 & & \\
\hline Alentejo & 8,4 & 3,5 & & \\
\hline Algarve & 7,9 & 4 & & \\
\hline Madeira & 13 & 3,6 & & \\
\hline Açores & 13,2 & 3,2 & & \\
\hline Instalações e requisitos de funcionamento & & & 35,1 & $<0,001$ \\
\hline Norte & 20,8 & 6 & & \\
\hline Centro & 19,5 & 5,3 & & \\
\hline Lisboa Vale do Tejo & 19,8 & 6,1 & & \\
\hline Alentejo & 23,5 & 4,5 & & \\
\hline Algarve & 14 & 7 & & \\
\hline Madeira & 19,6 & 4,9 & & \\
\hline Açores & 20,7 & 4,9 & & \\
\hline Formação e práxis no bloco operatório & & & 13,6 & 0,035 \\
\hline Norte & 11 & 2,9 & & \\
\hline Centro & 11,1 & 2,8 & & \\
\hline Lisboa Vale do Tejo & 11,5 & 2,8 & & \\
\hline Alentejo & 11,4 & 2,5 & & \\
\hline Algarve & 8,7 & 3,5 & & \\
\hline Madeira & 9,7 & 2,5 & & \\
\hline Açores & 10,9 & 2,9 & & \\
\hline Continuidade na assistência de enfermagem & & & 16,2 & 0,013 \\
\hline Norte & 4,2 & 2,8 & & \\
\hline Centro & 3,9 & 2,6 & & \\
\hline Lisboa Vale do Tejo & 4,1 & 2,7 & & \\
\hline Alentejo & 2,7 & 1,5 & & \\
\hline Algarve & 3,6 & 2,6 & & \\
\hline Madeira & 6 & 3,4 & & \\
\hline Açores & 3,4 & 1,8 & & \\
\hline
\end{tabular}


Especificidades dos grupos profissionais

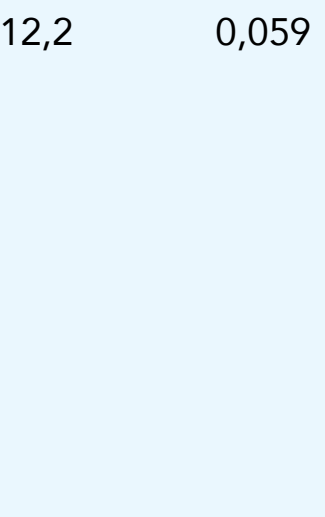

\begin{tabular}{lll}
\hline Norte & 3,8 & 1,2 \\
\hline Centro & 3,9 & 1,1 \\
\hline Lisboa Vale do Tejo & 4,1 & 0,9 \\
\hline Alentejo & 3,8 & 1,2 \\
\hline Algarve & 4,3 & 0,7 \\
\hline Madeira & 4,3 & 0,6 \\
\hline Açores & 3,6 & 1,3 \\
\hline
\end{tabular}

Dimensão Processo

Fluxos de comunicação

$17,3 \quad 0,008$

\begin{tabular}{lcc}
\hline Norte & 28,6 & 4,6 \\
\hline Centro & 27,9 & 4,5 \\
\hline Lisboa Vale do Tejo & 27,7 & 4,9 \\
\hline Alentejo & 28,8 & 3,4 \\
\hline Algarve & 25,3 & 7,6 \\
\hline Madeira & 29 & 1 \\
\hline Açores & 27 & 4,2
\end{tabular}

Estratégias de suporte aos processos de trabalho assistencial

\begin{tabular}{lll}
\hline Norte & 20,8 & 5,3 \\
\hline Centro & 19,9 & 4,7 \\
\hline Lisboa Vale do Tejo & 20,6 & 5,3 \\
\hline Alentejo & 19,9 & 4,6 \\
\hline Algarve & 17,5 & 5,6 \\
\hline Madeira & 22,3 & 1,5 \\
\hline Açores & 16,6 & 6,1 \\
\hline Controle para a segurança & & \\
\hline Norte & 9,1 & 1,5 \\
\hline Centro & 22,1 & 0,001 \\
\hline Lisboa Vale do Tejo & 9,4 & 1,2 \\
\hline Alentejo & 9,2 & 1,7 \\
\hline Algarve & 9,7 & 0,8 \\
\hline Madeira & 10 & 0 \\
\hline Açores & 10 & 0 \\
\hline Trabalho em equipe & 8,9 & 1,7 \\
\hline Norte & & \\
\hline Centro & 8,6 & 1,6 \\
\hline Lisboa Vale do Tejo & 8,7 & 1,6 \\
\hline Alentejo & 8,7 & 1,3 \\
\hline Algarve & 8,5 & 1,4 \\
\hline Madeira & 9,5 & 1,2 \\
\hline
\end{tabular}




\begin{tabular}{|c|c|c|c|c|}
\hline Açores & 9 & 1,9 & & \\
\hline \multicolumn{5}{|c|}{ Dimensão Resultado } \\
\hline Avaliação da qualidade dos cuidados & & & 42 & $<0,001$ \\
\hline Norte & 19,2 & 6,4 & & \\
\hline Centro & 17,9 & 6,3 & & \\
\hline Lisboa Vale do Tejo & 17,5 & 6,3 & & \\
\hline Alentejo & 17,1 & 5,8 & & \\
\hline Algarve & 15,3 & 6,2 & & \\
\hline Madeira & 21,7 & 2,5 & & \\
\hline Açores & 12,2 & 5,5 & & \\
\hline Avaliação dos processos & & & 37,2 & $<0,001$ \\
\hline Norte & 12,6 & 4,1 & & \\
\hline Centro & 11,3 & 3,9 & & \\
\hline Lisboa Vale do Tejo & 11,4 & 4,2 & & \\
\hline Alentejo & 11,9 & 3,4 & & \\
\hline Algarve & 9,5 & 4,2 & & \\
\hline Madeira & 15,3 & 3 & & \\
\hline Açores & 8,7 & 4,3 & & \\
\hline Sistemas de controle & & & 38,7 & $<0,001$ \\
\hline Norte & 10,3 & 3,3 & & \\
\hline Centro & 9,7 & 3,2 & & \\
\hline Lisboa Vale do Tejo & 10,3 & 3,3 & & \\
\hline Alentejo & 8,5 & 3,1 & & \\
\hline Algarve & 7,5 & 2,1 & & \\
\hline Madeira & 9 & 4,4 & & \\
\hline Açores & 8,8 & 3,7 & & \\
\hline
\end{tabular}

Fonte: Os autores (2019)

\section{DISCUSSÃO}

Os indicadores são tipicamente classificados em áreas específicas de cuidados, usando o modelo conceitual desenvolvido por Donabedian ${ }^{(15)}$. Estes indicadores avaliam as estruturas, os processos e os resultados dos cuidados de saúde e podem fornecer uma base quantitativa para a melhoria da qualidade.

Os indicadores de estrutura avaliam as configurações em que os cuidados de saúde ocorrem, incluindo recursos físicos, recursos humanos e estrutura administrativa ${ }^{(20)}$. $\mathrm{Na}$ estrutura estão as características estáveis e necessárias à prestação de cuidados, integrando recursos, estrutura física e organizacional, novas tecnologias, materiais, equipamentos adequados, entre outros ${ }^{(3,17,21)}$. 
Da aplicação do instrumento utilizado, no que se refere à sua estrutura, observa-se uma qualidade média na maioria dos fatores. Dos diferentes fatores da escala, salienta-se, pela negativa, o fator referente à "continuidade na assistência de enfermagem", cuja qualidade foi considerada baixa. Este fator reflete a importância das visitas pré e pós-operatórias realizadas pelo enfermeiro do centro cirúrgico. Com recurso a diferentes ferramentas de apoio, a enfermagem perioperatória permite um impacto positivo na satisfação do cliente, na segurança, na qualidade do atendimento e na economia de custos ${ }^{(17,22-23)}$. As visitas de avaliação pré e pós-operatória tornaram-se uma prática comum, aumentando a eficiência e a eficácia do atendimento no sentido humano e econômico ${ }^{(23)}$.

Ainda no âmbito da Estrutura, o fator "especificidades dos grupos profissionais", que foi considerado de alta qualidade, salienta a necessidade de uma gestão e coordenação adequada dos diferentes grupos profissionais para um melhor planejamento e eficiência das salas do centro cirúrgico(17). Os profissionais que trabalham no centro cirúrgico têm características diferentes e dividem responsabilidades que variam com as especialidades; perante a enorme variabilidade, a gestão deve ser feita de forma dinâmica e eficiente ${ }^{(3)}$.

Da mesma forma que são imprescindíveis os atributos da estrutura, também é importante a qualidade dos processos assistenciais, garantidos pela implementação de sistemas de melhoria contínua da qualidade, que influenciam o nível de cuidados cirúrgicos ${ }^{(6,24)}$.

No âmbito da avaliação do processo, foi relatada alta qualidade nos fatores "fluxos de comunicação", "controle para a segurança e "trabalho em equipe", cujos indicadores permitem melhorar a qualidade dos processos. Segundo Donabedian, até o que se faz bem é passível de ser melhorado(11). O fator "estratégias de suporte aos processos de trabalho assistencial" foi considerado de média qualidade, no qual se inclui, entre outras, a utilização de checklist como estratégia de qualidade e segurança, e que têm sofrido um forte incremento nos últimos anos ${ }^{(5,18)}$, associada ao investimento das políticas nacionais e internacionais de cirurgia segura.

Os indicadores de resultado permitem avaliar o impacto, refletindo as mudanças, no sentido desejável ou indesejável dos cuidados ${ }^{(5)}$. Hoje há uma forte preocupação com a identificação de indicadores, particularmente de resultados, que devem ficar para além dos classicamente utilizados na produção dos serviços essencialmente econômicos ${ }^{(19)}$.

No âmbito da dimensão resultado, os três fatores da escala foram considerados de média qualidade: avaliação da qualidade dos cuidados, dos processos e dos sistemas de controle. O resultado deve refletir as mudanças, no sentido desejável ou indesejável, porém a maioria das instituições seleciona diferentes combinações de métricas com base em requisitos e critérios locais, muitas das quais são familiares ${ }^{(19)}$. A dificuldade surge em determinar quais indicadores são mais importantes para a inclusão. Idealmente, os indicadores deveriam consistir em dados já disponíveis em sistemas de informação, prontamente mensuráveis, e medidas qualitativas ${ }^{(25)}$.

\section{Tipo de centro cirúrgico}

$\mathrm{Na}$ Tabela 2, observa-se as correlações da aplicação das escalas por tipo de atividade do bloco operatório. No que se refere ao tipo de centro cirúrgico, no âmbito da estrutura, com a única exceção do fator especificidades dos grupos profissionais, conclui-se que existem diferenças significativas em todos os fatores. Na aplicação da escala de avaliação do processo, observa-se que existem diferenças significativas apenas nos "fluxos de comunicação" e "estratégias de suporte aos processos de trabalho assistencial".

Da aplicação da escala sobre os resultados, observam-se diferenças significativas em todos os fatores. Estas diferenças colocam em todos os itens os blocos operatórios ambulatórios com melhor qualidade, seguindo-se os centrais e por último os blocos de urgência. Esta assimetria por tipologia de bloco operatório vai ao encontro do relatório 
sobre a avaliação da situação nacional dos blocos operatórios realizada pelo Ministério da Saúde em 2015, em que, pela análise de alguns indicadores, verificou-se que existem grandes variações entre os diferentes tipos de Blocos Operatórios ${ }^{(6)}$.

A supremacia dos centros cirúrgicos de ambulatório vai ao encontro do referido por estes autores, os quais referem que os indicadores de qualidade portugueses para a cirurgia de ambulatório estão bem adaptados às atuais práticas internacionais ${ }^{(26)}$. Este aspecto pode estar relacionado com o forte incremento dado pelo Sistema Nacional de Saúde Português no desenvolvimento da cirurgia de ambulatório.

\section{Avaliação por região}

Num estudo realizado para avaliar a eficiência de unidades hospitalares em Portugal, com resultados de 27 hospitais, os autores evidenciaram disparidades que deveriam merecer atenção especial dos formuladores de políticas e gerentes de hospitais ${ }^{(16)}$. Este aspecto também foi evidenciado neste estudo.

$\mathrm{Na}$ avaliação da estrutura, com a única exceção em "especificidades dos grupos profissionais", concluiu-se que existem diferenças significativas em todos os outros fatores. Já no âmbito da avaliação do processo, a única exceção foi "trabalho em equipe", e na aplicação da escala sobre o resultado, notou-se diferenças significativas em todos os fatores. Tais dados concordam com o relatório de avaliação da situação nacional dos blocos operatórios, tanto na sua tipologia, quanto por região. Este relatório realça que se deve incentivar a otimização dos centros cirúrgicos, propondo o desenvolvimento de um benchmarking periódico de monitorização que permita identificar desajustes e incoerências e promover os casos de boas práticas em eficiência, qualidade e segurança ${ }^{(6)}$.

Sobre este percurso, importa realçar que a estrutura, processo e estrutura de resultados foram descritos pela primeira vez por Donabedian, que procurou dar um vocabulário para entender o que muitos começaram a intuir: o fato de que alguns doentes parecem ter melhores cuidados do que outros ${ }^{(24)}$. Desde então, tornou-se uma referência na melhoria da qualidade dos cuidados de saúde, com a preconização da sua utilização na segurança em centro cirúrgico.

Não é possível alcançar a excelência sem observar e atuar sobre o sistema de saúde. Isso demonstra, para além do que Donabedian antecipou, o valor de uma melhor compreensão científica dos cuidados de saúde como um sistema, e da importância da definição e redefinição contínua dos processos de cuidados, que permitam a melhoria contínua ${ }^{(25)}$.

Como limitação deste estudo, considera-se o fato de se restringir a uma parte de todas as instituições nacionais, tendo sido a coleta de dados realizada pelos profissionais que lá atuam. As avaliações são muito dísparas em cada uma das dimensões e nos diferentes fatores das escalas. Das diversas relações analisadas, não foi possível quantificar a qualidade do bloco operatório por região, dada a diversidade de discrepâncias nos diferentes fatores das escalas utilizadas.

\section{CONCLUSÃO}

Sobre este estudo, salienta-se que, dos 71 hospitais incluídos, nos diversos fatores das escalas, a avaliação é na sua maioria média, sendo considerada baixa no fator "continuidade na assistência de enfermagem". Em oposição, realça-se que os fatores "especificidades dos grupos profissionais", "fluxos de comunicação", "controle para a segurança" e "trabalho em equipe" foram considerados de alta qualidade. 
Este estudo aponta para a existência de desigualdades por tipologia de bloco e por região. A realização deste tipo de pesquisa, destinada a avaliar a qualidade do centro cirúrgico nas categorias de estrutura, processo e resultado, permite identificar lacunas que podem ser melhoradas e que exigem maior atenção. A garantia da qualidade em sáude requer o comprometimento integral e incondicional de todos os profissionais que atuam no setor, onde se afirma o papel de destaque da enfermagem.

Este estudo permitiu identificar áreas com necessidade de melhoria, constituindo uma ferramenta valiosa para utilização em ambiente hospitalar ou em investigação, e passível de ser replicada em estudos futuros. A identificação destes achados assume especial relevância para a enfermagem, pois os enfermeiros participam ativamente nos âmbitos decisórios, estratégicos e assistenciais de saúde.

\section{REFERÊNCIAS}

1. Gómez-Ríos MA, Abad-Gurumeta A, Casans-Francés R, Calvo-Vecino JM. Keys to optimize operating room efficiency. Rev Esp Anestesiol Reanim. [Internet]. 2019 [acesso em 11 dez 2019]; 66(2). Disponível em: https://doi.org/10.1016/j.redare.2018.08.011.

2. Perkins JN, Chiang T, Ruiz AG, Prager JD. Auditing of operating room times: a quality improvement project. Int J Pediatr Otorhinolaryngol. [Internet]. 2014 [acesso em $11 \mathrm{dez}$ 2019]; 78(5). Disponível em: https://doi.org/10.1016/j.ijporl.2014.02.010.

3. Costa Junior A da S, Leão LEV, Novais MAP de, Zycchi P. An assessment of the quality indicators of operative and non-operative times in a public university hospital. Einstein. [Internet]. 2015 [acesso em 08 dez 2019]; 13(4). Disponível em: http://dx.doi.org/10.1590/S1679-45082015GS3289.

4. Gabriel RA, Gimlich R, Ehrenfeld JM, Urman RD. Operating room metrics score card-creating a prototype for individualized feedback. J Med Syst [Internet]. 2014 [acesso em 11 dez 2019]; 38(144). Disponível em: http://dx.doi.org/10.1007/s10916-014-0144-8.

5. Gomes JAP, Martins MM, Fernandes CSN da N. Instrumentos para avaliar a qualidade e segurança no bloco operatório - revisão integrativa. Cogitare enferm. [Internet]. 2016 [acesso em 08 dez 2019]; 21(5). Disponível em: http://dx.doi.org/10.5380/ce.v21i5.45640.

6. Ministério da Saúde (PT). Avaliação da situação nacional dos blocos operatórios - Relatório Final. [Internet]. 2015 [acesso em 08 dez 2019]. Disponível em: http://www.apca.com.pt/documentos/2015/ Avaliacao situacao nacional blocos operatorios Outubro2015.pdf.

7. Wu Q, Huang LH, Xing MY, Feng ZX, Shao LW, Zhang MY, et al. Establishing nursing-sensitive quality indicators for the operating room: a cross-sectional delphi survey conducted in China. Australian Critical Care. [Internet]. 2017 [acesso em 08 dez 2019]; 30(1). Disponível em: http://dx.doi.org/10.1016/j. aucc.2016.04.003.

8. Joseph A, Bayramzadeh S, Zamani Z, Rostenberg B. Safety, Performance, and satisfaction outcomes in the operating room: a literature review. Herd. [Internet]. 2018 [acesso em 08 dez 2019]; 11(2). Disponível em: http://dx.doi.org/10.1177/1937586717705107.

9. Govaert JA, Bommel ACM van, Dijk WA van, Leersum NJ van, Tollenaar RAEM, Wouters MWJM. Reducing healthcare costs facilitated by surgical auditing: a systematic review. World J Surg. [Internet]. 2015 [acesso em 11 dez 2019]; 39. Disponível em: http://dx.doi.org/10.1007/s00268-015-3005-9.

10. Zhao P, Li Y, Li Z, Jia P, Zhang L, Zhang M. Use of patient safety culture instruments in operating rooms: a systematic literature review. J Evid Based Medic. [Internet]. 2017 [acesso em 11 dez 2019]; 10(2). Disponível em: https://doi.org/10.1111/jebm.12255. 
11. Berwick D, Fox DM. Evaluating the quality of medical care: Donabedian's classic article 50 years later. Milbank Quaterly. [Internet]. 2016 [acesso em 11 dez 2019]; 94(2). Disponível em: http://dx.doi. org/10.1111/1468-0009.12189.

12. Donabedian, A. Uma introdução à garantia de qualidade nos cuidados de saúde. New York: Oxford University Press; 2003.

13. Ayanian JZ, Markel H. Donabedian's lasting framework for health care quality. N Engl J Med. [Internet]. 2016 [acesso em 11 dez 2019]; 375. Disponível em: http://dx.doi.org/10.1056/NEJMp1605101.

14. World Health Organization (WHO). Checklists save lives. Bull World Health Organ. [Internet]. 2008 [acesso em 08 dez 2019]; 86(7). Disponível em: https://www.who.int/bulletin/volumes/86/7/08-010708. pdf?ua=1.

15. Simões J de A, Augusto GF, Fronteira I, Hernández-Quevedo C. Portugal: health system review. Health Syst Transit. [Internet]. 2017 [acesso em $11 \mathrm{dez}$ 2019];19(2). Disponível em: http://ihmtweb.ihmt. unl.pt/PublicacoesFB/HiT-Portugal-EN/files/assets/common/downloads/Health\%20Systems\%20in\%20 Transition\%20-\%20Portugal.pdf.

16. Ferreira DC, Nunes AM. Technical efficiency of portuguese public hospitals: a comparative analysis across the five regions of Portugal. Int J Health Plann Manag. [Internet]. 2018 [acesso em 11 dez 2019]; 34(1). Disponível em: http://dx.doi.org/10.1002/hpm.2658.

17. Gomes JA, Martins MM, Tronchin DMR, Fernandes CS. Validation of a scale on structure indicators in the operating room: contributions to nursing. Aquichan. [Internet]. 2019 [acesso em $11 \mathrm{fev} 2020$ ]; 19(1). Disponível em: https://aquichan.unisabana.edu.co/index.php/aquichan/article/view/9613.

18. Gomes JA, Martins MM, Tronchin DMR, Fernandes CS. Processos de qualidade assistencial no bloco operatório - validação de uma escala. J Health NPEPS. [Internet]. 2018 [acesso em 11 dez 2019]; 3(2). Disponível em: http://dx.doi.org/10.30681/252610103118.

19. Gomes JA, Martins MM, Tronchin DMR, Fernandes CS. Validation of an instrument to measure the results of quality assistance in the operating room. Q Manag Health Care [Internet]. 2019 [acesso em 11 fev 2020]; 28(2). Disponível em: http://dx.doi.org/10.1097/QMH.0000000000000205.

20. Chazapis M, Gilhooly D, Smith AF, Myles PS, Haller G, Grocott MPW, et al. Perioperative structure and process quality and safety indicators: a systematic review. $\mathrm{Br}$ J Anaesth. [Internet]. 2018 [acesso em $11 \mathrm{dez}$ 2019]; 120(1). Disponível em: http://dx.doi.org/10.1016/j.bja.2017.10.001.

21. Fernandes HM de LG, Peniche $A$ de CG. Perceção da equipe de enfermagem do centro cirúrgico acerca da Acreditação Hospitalar em um Hospital Universitário. Rev Esc Enferm USP. [Internet]. 2015 [acesso em 11 dez 2019]; 49(spe). Disponível em: http://dx.doi.org/10.1590/S0080-623420150000700004.

22. Gürsoy A, Candas B, Güner S, Yilmaz S. Preoperative stress: an operating room nurse intervention assessment. J Perianesth Nurs. [Internet]. 2016 [acesso em 11 dez 2019]; 31(6). Disponível em: http:// dx.doi.org/10.1016/j.jopan.2015.08.011.

23. Turunen E, Miettinen M, Setälä L, Vehviläinen-Julkunen K. An integrative review of a preoperative nursing care structure. J Clin Nurs. [Internet]. 2017 [acesso em 11 dez 2019]; 26(7-8). Disponível em: https://pubmed.ncbi.nlm.nih.gov/27325370/.

24. Brownlee SA, Whitson PJ, Ibrahim AM. Measuring and improving the design quality of operating rooms. Surg Infect. (Larchmt). [Internet]. 2019 [acesso em 11 fev 2020]; 20(2). Disponível em: https:// pubmed.ncbi.nlm.nih.gov/30789799/.

25. Fixler T, Wright JG. Identification and use of operating room efficiency indicators: the problem of definition. J can chir. [Internet]. 2013 [acesso em 11 fev 2019]; 56(4). Disponível em: http://dx.doi. org/10.1503/cjs.020712.

26. Nunes JS, Gomes R, Povo A, Alves EC. Quality indicators in ambulatory surgery: a literature review comparing portuguese and international systems. Acta Medica Port. [Internet]. 2018 [acesso em $11 \mathrm{fev}$ 
2020]; 31(7-8). Disponível em: http://dx.doi.org/10.20344/amp.10416.

\section{COMO REFERENCIAR ESTE ARTIGO:}

Gomes JA, Martins MM, Tronchin D, Fernandes CS. Avaliação da qualidade do centro cirúrgico na estrutura, processo e resultados. Cogitare enferm. [Internet]. 2021 [acesso em "colocar data de acesso, dia, mês abreviado e ano"]; 26. Disponível em: http://dx.doi.org/10.5380/ce.v26i0.71083.

*Artigo extraído da tese de doutorado "A Qualidade Assistencial no Bloco Operatório de Hospitais Portugueses". Instituto de Ciências Biomédicas Abel Salazar, Portugal, 2020.

Recebido em: 04/01/2020

Aprovado em: 05/10/2020

Autor Correspondente:

Carla Silvia Fernandes

Escola Superior de Enfermagem do Porto - Porto, Portugal

E-mail: carlasilviaf@gmail.com

Contribuição dos autores:

Contribuições substanciais para a concepção ou desenho do estudo; ou a aquisição, análise ou interpretação de dados do estudo - JAG, CSF

Aprovação da versão final do estudo a ser publicado - MMM, DT

Copyright () 2021 Este é um artigo em acesso aberto distribuído nos termos da Licença Creative Commons Atribuição, que permite o uso irrestrito, a distribuição e reprodução em qualquer meio desde que $\circ$ artigo original seja devidamente citado. 